\title{
Correction to: Male-to-Female Breast Augmentation and Body Contouring
}

\author{
Stelios C. Wilson, Shane D. Morrison,
}

Scott W. Mosser, and Thomas Satterwhite

\section{Correction to: \\ Chapter 10 in: L. S. Schechter (ed.), Gender Confirmation Surgery, https://doi.org/10.1007/978-3-030-29093-1_10}

Owing to an oversight on the part of the authors of this chapter the authorgroup were initially published with errors. The correct presentation is given here. This correction (Authorship) has been updated in book.

Stelios C. Wilson, Shane D. Morrison, Scott W. Mosser, and Thomas Satterwhite

S. C. Wilson

Hansjorg Wyss Department of Plastic Surgery, New York University School of Medicine, New York, NY, USA

e-mail: Stelios.Wilson@nyumc.org

\section{S. D. Morrison}

Division of Plastic Surgery, Department of Surgery, University of Washington Medical Center, Seattle, WA, USA

e-mail: shanedm@uw.edu

\section{S. W. Mosser}

Saint Francis Memorial Hospital, San Francisco, CA, USA

e-mail: swmosser@drmosser.com

T. Satterwhite $(\square)$

Align Surgical Associates, Inc, San Francisco, CA, USA

The updated version of this chapter can be found at

https://doi.org/10.1007/978-3-030-29093-1_10 